\title{
Microtubule Plus-End Tracking Proteins SLAIN1/2 and ch-TOG Promote Axonal Development
}

\author{
Babet van der Vaart, ${ }^{1 \star}$ Mariella A. M. Franker, ${ }^{2 \star}$ Marijn Kuijpers, ${ }^{2}$ Shasha Hua, ${ }^{2}$ Benjamin P. Bouchet, ${ }^{2}$ Kai Jiang, ${ }^{2}$ \\ Ilya Grigoriev, ${ }^{2}$ Casper C. Hoogenraad, ${ }^{2}$ and Anna Akhmanova ${ }^{2}$ \\ ${ }^{1}$ Department of Cell Biology, Erasmus Medical Center, 3000 CA Rotterdam, The Netherlands, and ${ }^{2}$ Cell Biology, Faculty of Science, Utrecht University, 3584 \\ CH Utrecht, The Netherlands
}

Development, polarization, structural integrity, and plasticity of neuronal cells critically depend on the microtubule network and its dynamic properties. SLAIN1 and SLAIN2 are microtubule plus-end tracking proteins that have been recently identified as regulators of microtubule dynamics. SLAINs are targeted to microtubule tips through an interaction with the core components of microtubule plus-end tracking protein network, End Binding family members. SLAINs promote persistent microtubule growth by recruiting the microtubule polymerase ch-TOG to microtubule plus-ends. Here, we show that SLAIN1/2 and ch-TOG-proteins are highly enriched in brain and are expressed throughout mouse brain development. Disruption of the SLAIN-ch-TOG complex in cultured primary rat hippocampal neurons by RNA interference-mediated knockdown and a dominant-negative approach perturbs microtubule growth by increasing catastrophe frequency and inhibits axon extension during neuronal development. Our study shows that proper control of microtubule dynamics is important for axon elongation in developing neurons.

\section{Introduction}

Neurons are polarized cells, which critically depend on the cytoskeleton for their development and maintenance. Neuronal microtubules (MTs) play a pivotal role in controlling cell shape, actin organization, and the polarized distribution of proteins, vesicles, and other organelles (Conde and Cáceres, 2009; Hoogenraad and Bradke, 2009; Poulain and Sobel, 2010).

In mature neurons, both axons and dendrites contain bundles of highly stabilized MTs that are cross-linked by the MTassociated proteins (MAPs). At the same time, a proportion of MTs remains dynamic and is crucial for the functional and structural plasticity of neurons (Conde and Cáceres, 2009; Hoogenraad and Bradke, 2009; Poulain and Sobel, 2010). Dynamic MTs are especially important in developing neurons, and even moderate changes in MT stability can affect neuronal polarity (Witte et al., 2008).

An important group of factors that regulate different aspects of MT dynamics are MT plus-end tracking proteins (+TIPs), which associate specifically with growing MT plus-ends (Schuy-

\footnotetext{
Received March 13, 2012; revised Aug. 23, 2012; accepted Aug. 24, 2012

Author contributions: B.v.d.V., C.C.H., and A.A. designed research; B.v.d.V., M.A.M.F., M.K., S.H., B.P.B., K.J., and I.G. performed research; B.v.d.V., M.A.M.F., M.K., S.H., B.P.B., K.J., and I.G. analyzed data; B.v.d.V., M.A.M.F., C.C.H., and A.A. wrote the paper.

This work was supported by the Netherlands Organization for Scientific Research (NWO) Grants NWO-ALW-VICI, ZonMw TOP, Open Program, and Foundation for Fundamental Research on Matter (FOM) Program to A.A. and C.C.H. We thank L. Cassimeris for the ch-TOG antibody; and N. Keijzer, P. Wulf, and V. van Dis for the help with primary neuron cultures and tissue extracts.

*B.v.d.V. and M.A.M.F. contributed equally to this manuscript.

Correspondence should be addressed to either Anna Akhmanova or Casper C. Hoogenraad, Cell Biology, Faculty of Science, Utrecht University, Padualaan 8, 3584 CH, Utrecht, The Netherlands. E-mail: a.akhmanova@uu.nl or c.hoogenraad@uu.nl.

DOI:10.1523/JNEUROSCI.1240-12.2012

Copyright $\odot 2012$ the authors $\quad 0270-6474 / 12 / 3214722-07 \$ 15.00 / 0$
}

ler and Pellman, 2001; Akhmanova and Steinmetz, 2008). End Binding (EB) proteins are considered to be core + TIP complex components as they can autonomously track growing MT plusends and target to them many other + TIPs. In mammals, there are three EB family members: EB3 is a brain-enriched protein, whereas EB1 and EB2 are more widely expressed (Nakagawa et al., 2000; Jaworski et al., 2009).

Recently, we identified the SLAIN family as novel + TIPs that interact with EBs (van der Vaart et al., 2011). In mammals, two SLAIN homologues exist, SLAIN1 and SLAIN2 (Hirst et al., 2006). We showed that in nonneuronal cells, SLAIN2 is an EBdependent + TIP that also interacts with three other + TIP families: the CLIPs, CLASPs, and XMAP215/ch-TOG (van der Vaart et al., 2011). By binding to multiple + TIPs, SLAIN2 can promote formation of + TIP interaction networks. This property enables SLAIN2 to enhance the accumulation of the MT polymerase chTOG at the growing MT plus-ends and in this way stimulate persistent MT growth (van der Vaart et al., 2011).

Although neurons are postmitotic cells, they often employ molecular machinery used in dividing cells (Baas, 1999). ch-TOG is the only mammalian member of the XMAP215 family of MT polymerases that is generally assumed to be directly responsible for the high rates of MT polymerization in vivo compared with in vitro systems (Howard and Hyman, 2007). Despite the potentially crucial role of ch-TOG in controlling MT dynamics, its function has not been investigated in neuronal cells.

In this study, we addressed this gap in understanding of regulation of MT dynamics in neurons. We showed that both chTOG and SLAIN1/2 are highly expressed in mammalian brain and in cultured neurons. Disruption of the SLAIN-ch-TOG complex affected MT growth by increasing catastrophe frequency and led to a decrease in axon length. We conclude that the SLAIN-ch- 
TOG complex plays an important role in axon outgrowth. This study provides new molecular insight into the role of proper regulation of MT dynamics in neuronal development.

\section{Materials and Methods}

Constructs. $\mathrm{p} \beta$ actin-HA- $\beta$-galactosidase has been previously described (Hoogenraad et al., 2005); GFP-SLAIN2-N1 (van der Vaart et al., 2011) was cloned into neuronal GFP-GW1 expression vector (modified GW1vector; British Biotechnology) (Hoogenraad et al., 2005). The CherryMT+TIP construct contains the two-stranded leucine zipper coiled-coil sequence corresponding to GCN4-p1 (RMKQLEDKVEELLSKNYHLENEVARLKKLVGER) fused to a glycine rich-linker sequence (GAGG) followed by the C-terminal 43 aa peptide ETVPQTHRPTPRAGSRPSTAKPSKIPTPQRKSPASKLDKSSKR of human MACF2 (E5455R5497; NP_899236), which binds to EB1 and tracks growing MT ends in an EB1-dependent manner (Honnappa et al., 2009). The construct was inserted in pBactin-16-pl expression vector. pSuper-based shRNA vectors were directed against the following target sequences: mouse/rat chTOG AGAGTCCAGAATGGTCCAA, rat/human SLAIN1 ATCTAT CGACAGTGAGCTG; rat SLAIN2 CTCTATAGATAGTGAGTTA (van der Vaart et al., 2011). shRNA lentiviral constructs were created by inserting the $\mathrm{H} 1$ promoter and shRNA sequences described above into the $\mathrm{XbaI}$ and XhoI sites of pLentiLox3.7, which contained EGFP driven by a human synapsin 1 promoter (Rubinson et al., 2003; Nakagawa and Hoogenraad, 2011).

Neuronal cultures, transfection, and lentiviral transduction. Primary hippocampal cultures were prepared from brains of E18 rats of either sex as described by Jaworski et al. 2(009) and transfected using Lipofectamine 2000 (Invitrogen) for live imaging and immunofluorescent staining. To generate Western blots of shRNA-expressing neurons, neuronal cultures were transduced with lentiviruses as described previously (Rubinson et al., 2003; Nakagawa and Hoogenraad, 2011).

Antibodies, immunofluorescent cell staining, immunoprecipitation, and Western blotting. Neurons were fixed with $4 \%$ PFA/4\% sucrose in PBS for $10 \mathrm{~min}$ at room temperature to visualize neuronal morphology, and with a combination of cold methanol and paraformaldehyde to stain for EB1, ch-TOG, or SLAIN1/2. Extracts of tissues and primary hippocampal neuron cultures and immunoprecipitation were done as described previously (Jaworski et al., 2009; van der Vaart et al., 2011).

We used rabbit polyclonal antibodies against SLAIN1/2 (van der Vaart et al., 2011), $\beta$-galactosidase (MP Biomedicals), and ch-TOG (Charrasse et al., 1998) (a gift from L. Cassimeris, Lehigh University, Bethelehem, PA); mouse monoclonal antibodies against EB1 (BD Biosciences), MAP2 (Santa Cruz Biotechnology), $\beta$-tubulin, tau, and actin (Millipore Bioscience Research Reagents). The following secondary antibodies were used: alkaline phosphatase-conjugated anti-rabbit; anti-mouse or anti-rat antibodies (Sigma-Aldrich); IRDye 800CW goat anti-rabbit, anti-mouse, and anti-rat IgG (Li-Cor Biosciences); Alexa-350-, Alexa-488-, and Alexa-598-conjugated goat antibodies against rabbit, rat, and mouse IgG (Invitrogen).

Image acquisition and processing. Images of fixed cells were collected with a Leica DMRBE microscope equipped with a PL Fluotar $100 \times 1.3$ N.A. or $40 \times 1.00-0.50$ N.A. oil objective, FITC/EGFP filter 41012 (Chroma) and Texas Red filter 41004 (Chroma), and an ORCA-ER-1394 CCD camera (Hamamatsu) or with a Nikon A1R confocal laser microscope equipped with a $20 \times$ air Plan-Apo N.A. 0.75 , a $40 \times$ oil Plan-Fluor N.A. 1.3, and a $60 \times$ oil Plan-Apo N.A. 1.4 objective.

Time-lapse live-cell imaging of Cherry-MT+ TIP was performed on a Nikon Eclipse TE2000E equipped with an incubation chamber (INUG2ZILCS-H2; Tokai Hit) mounted on a motorized stage (Prior). Coverslips were mounted in metal rings, immersed in conditioned medium, and maintained at $37^{\circ} \mathrm{C}$ and $5 \% \mathrm{CO}_{2}$. To image fast MT dynamics, we performed two color total internal reflection fluorescence (TIRF) microscopy using a Nikon microscope equipped with a $100 \times$ Apo TIRF N.A. 1.49 objective, $2.5 \times$ Optovar and EMCCD camera (QuantEM; Roper Scientific). For excitation and imaging of GFP, we used $488 \mathrm{~nm}$ argon laser (Spectra-Physics Lasers) in combination with ET-GFP filter set (49002; Chroma) and for mCherry we used $561 \mathrm{~nm}$ diode-pumped solid- state laser (Melles Griot) combined with Cherry ET-mCherry filter set (49008; Chroma).

Image processing, kymograph analysis, and various quantifications were performed using MetaMorph (Molecular Devices). Analysis of neuron morphology was performed using SynD (Schmitz et al., 2011). Images were prepared for publication using MetaMorph and Adobe Photoshop. Statistical analysis was performed using nonparametric Mann-Whitney $U$ test in Statistica for Windows and SigmaPlot.

\section{Results}

\section{Expression of ch-TOG and SLAIN/2 in brain}

Although initially identified in tumors, hence its name, colonic and hepatic tumor-overexpressed gene (ch-TOG), ch-TOG is ubiquitously expressed in healthy tissues, with elevated levels in the brain (Charrasse et al., 1995, 1996). Using reverse-transcriptase PCR (RT-PCR), we have previously shown that both SLAIN1 and SLAIN2 are expressed in human brain (van der Vaart et al., 2011). Based on a gene trap mouse-line and in situ hybridization, SLAIN1 is predominantly expressed in the developing nervous system (Hirst et al., 2006, 2010).

Using Western blot analysis, we found that ch-TOG is highly enriched in a variety of different brain regions (Fig. $1 A$ ), in line with previous RT-PCR results (Charrasse et al., 1995, 1996). This expression pattern correlates with the high levels of $\beta$-tubulin found in these regions. Using a pan-SLAIN antibody that recognizes both SLAIN1 and SLAIN2 equally well (van der Vaart et al., 2011), we observed multiple positive bands that likely correspond to different splice isoforms and/or posttranslationally modified forms of SLAIN1/2 in the different tissues tested (Fig. 1A). In different brain regions, the double band at $\sim 80 \mathrm{kDa}$ detected with SLAIN1/2 antibodies (Fig. $1 A$ ) is similar to nonphosphorylated SLAIN2 seen in nonneuronal interphase cells (van der Vaart et al., 2011).

Next, we looked in more detail at ch-TOG and SLAIN1/2 expression levels in different mouse brain regions during brain development. We observed constant levels of ch-TOG, while SLAIN1/2 expression was somewhat reduced in mature tissues (Fig. $1 B$ ). These data indicate that ch-TOG and SLAIN1/2 are expressed in the brain and suggest that SLAINs might be more important at early developmental stages, in agreement with the data on SLAIN1 transcription (Hirst et al., 2006, 2010).

We tested whether SLAIN1/2 and ch-TOG associate with each other in brain tissues and found that this was indeed the case: ch-TOG coprecipitated with SLAIN1/2 from extracts of embryonic cortex and adult brain (Fig. 1C), similar to dividing cultured cells (van der Vaart et al., 2011). Coprecipitation of the two proteins was somewhat weaker from the adult tissue, suggesting that the complex might be more prominent during development.

To investigate the role of the SLAIN-ch-TOG complex in neuronal development, we made use of rat hippocampal neuronal cultures undergoing terminal differentiation (Banker and Goslin, 1988). In neuronal cultures, SLAIN1/2 and ch-TOG were present at relatively constant levels throughout development (Fig. 1D). Using immunofluorescent labeling, endogenous ch-TOG and SLAIN1/2 could clearly be detected in cell body, neurites, and axonal and dendritic growth cones of neurons at day 5 in vitro (DIV5) (Fig. 1E,F). Colocalization of SLAIN1/2 and ch-TOG with the plus-end marker EB1 was especially clear in the growth cones (Fig. $1 E, F$ ), which are the sites where highly dynamic MTs interact with the actin cytoskeleton to steer neurite growth (Conde and Cáceres, 2009; Hoogenraad and Bradke, 2009; Poulain and Sobel, 2010). 
A

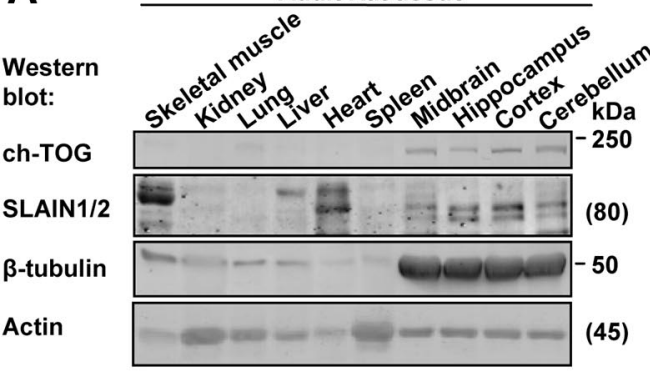

B

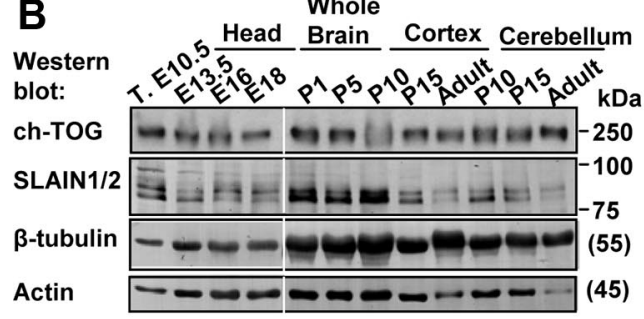

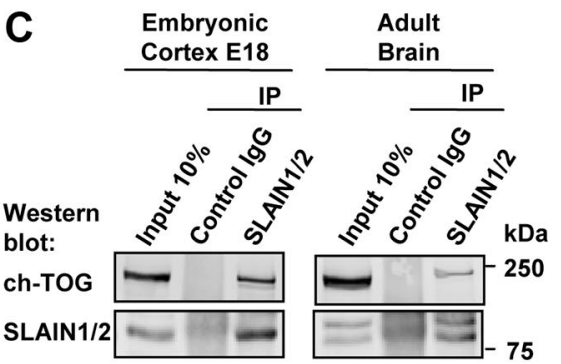

D

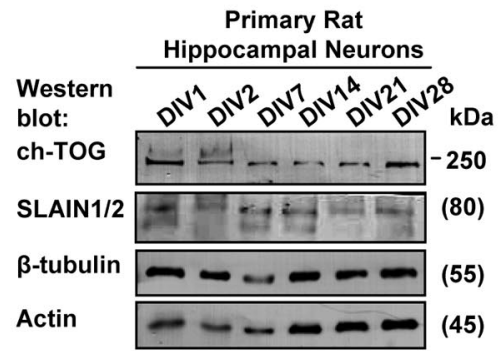

$\mathbf{E}$

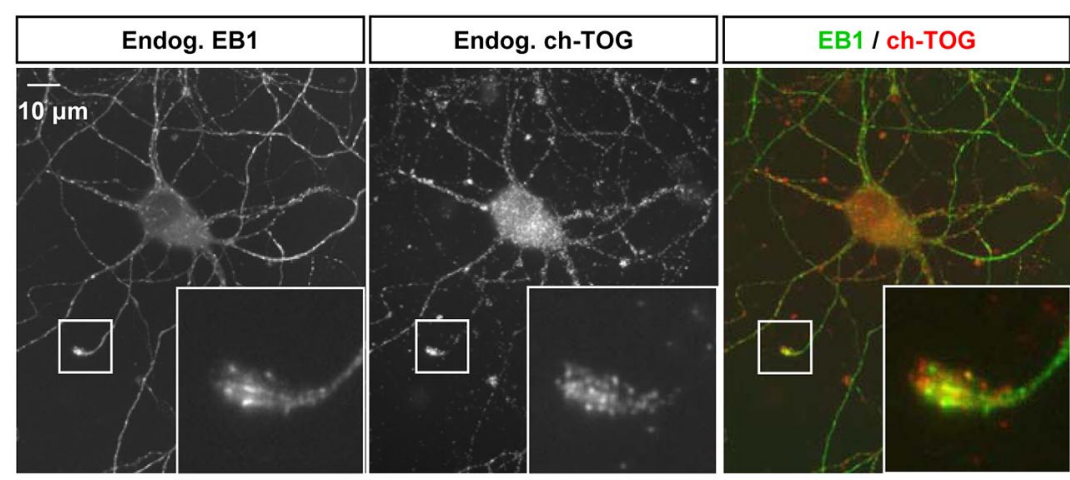

$\mathbf{F}$

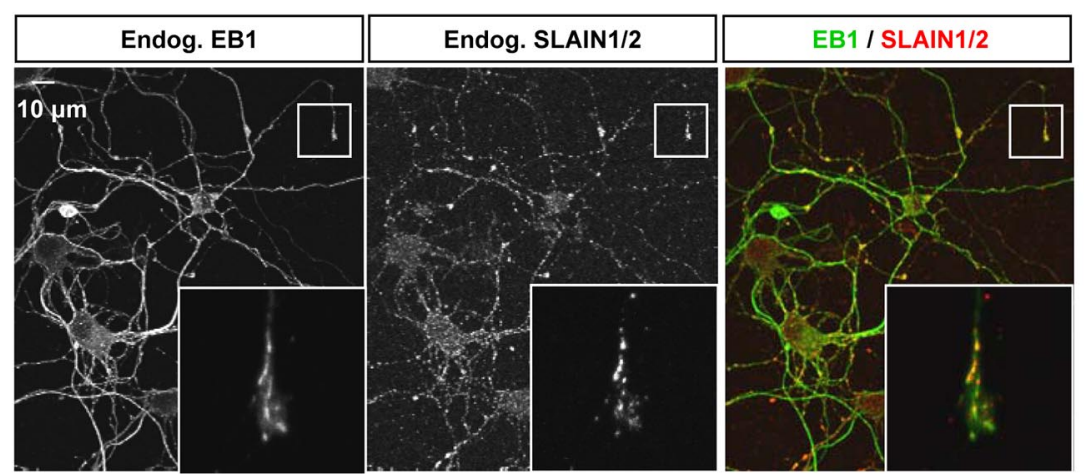

Figure 1. ch-TOG and SLAIN1/2 are expressed and interact with each other in brain tissue. $\boldsymbol{A}, \boldsymbol{B}, \boldsymbol{D}$, Western blot analysis of different adult rat tissues $(\boldsymbol{A})$, mouse brain tissue of different developmental stages $(\boldsymbol{B})$, and extracts of primary hippocampal neuron culture extracts at different stages of development in vitro (D) using the indicated antibodies. $\boldsymbol{C}$, Immunoprecipitations (IP) from extracts of rat embryonic cortex or adult brain with either control IgG or SLAIN1/2 antibodies analyzed by Western blotting with the indicated antibodies. $E$, $F$, Images of neurons fixed on DIV5 and labeled with the indicated antibodies. The insets show enlargements of the boxed areas, which show a dendritic $(\boldsymbol{E})$ and an axonal $(\boldsymbol{F})$ growth cone. In the overlay, EB1 is shown in green and ch-TOG $(\boldsymbol{E})$ and SLAIN1/2 $(\boldsymbol{F})$ in red. Endog., Endogenous; T., total.

Disruption of the SLAIN-ch-TOG complex affects MT growth in neurons

To assess the function of the SLAIN-ch-TOG complex during neuronal development, it was disrupted in two different ways. First, we performed RNA interference-mediated knockdown of SLAIN1/2 and ch-TOG using previously described shRNAs against ch-TOG and SLAIN2 (van der Vaart et al., 2011). SLAIN1 target sequence was chosen in the region homologous to that of SLAIN2 shRNA. The efficiency of protein depletion was tested by introducing shRNAs into neuronal cultures by lentiviral transduction. Single SLAIN1 and SLAIN2 depletions had only a mild effect on the SLAIN1/2 pool (data not shown), while a combination of SLAIN1 and SLAIN2 shRNAs reduced this pool by $\sim 60 \%$ (Fig. $2 A$ ); ch-TOG was depleted by $\sim 80 \%$ (Fig. $2 A$ ). The lower efficiency of SLAIN1/2 depletion was likely due to the fact that the two proteins had to be depleted simultaneously.

As an alternative approach, the SLAIN-ch-TOG complex was disrupted by overexpression of a dominant-negative SLAIN2 
A
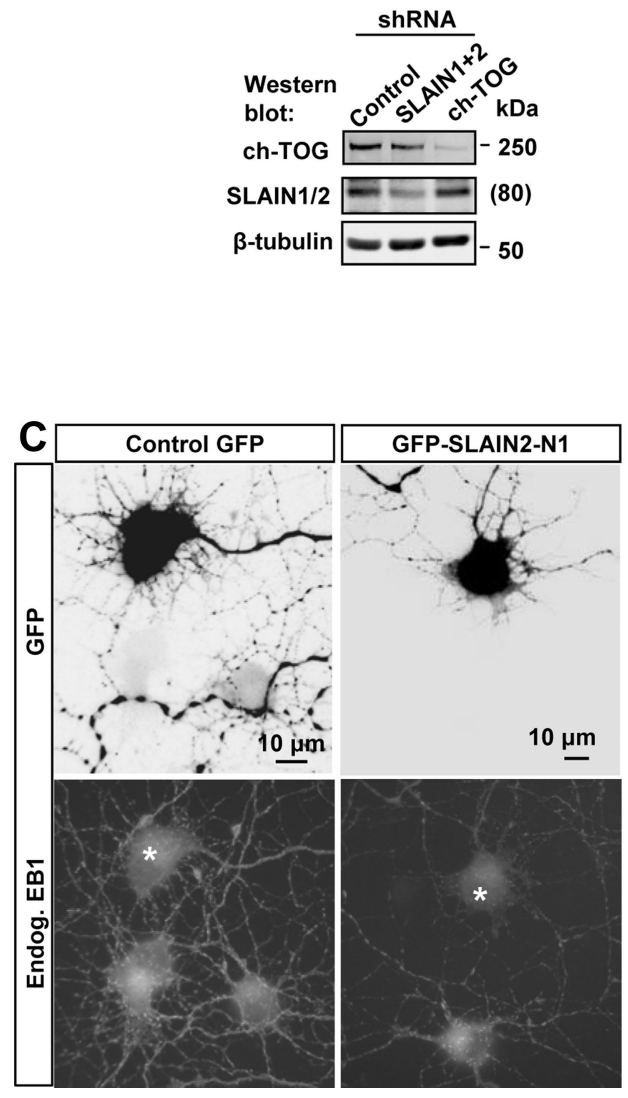

B

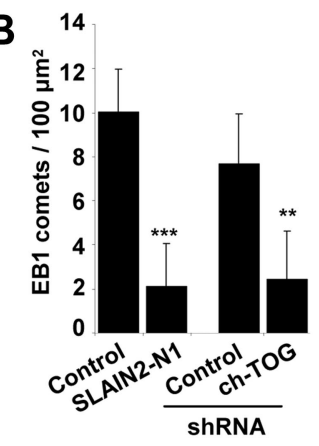

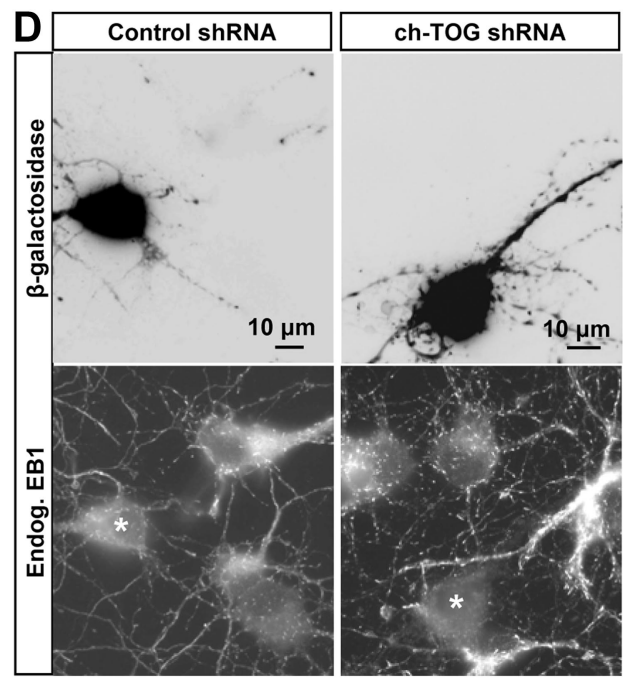

Figure 2. Disruption of the SLAIN-ch-TOG complex in neuronal cells. $\boldsymbol{A}$, Western blots of extracts of hippocampal neuronal cultures transduced with lentiviruses expressing the indicated shRNAs. SLAIN1 + 2 - simultaneous transduction with shRNAs against SLAIN1 and SLAIN2. B, Quantification of the number of EB1 comets in the cell body per $100 \mu m^{2}{ }^{2}$ surface area in control shRNA, ch-TOG shRNA, GFP control, and GFP-SLAIN2-N1-expressing neurons (10-15 cells were analyzed for each condition). Statistically significant differences are indicated ( $\left.{ }^{* *} p<0.05,{ }^{* * *} p<0.001\right)$. Error bars indicate SD. C, D, Images of neurons transfected at DIV1 with indicated constructs, fixed on DIV5, and labeled with antibodies against EB1. Asterisks in $\boldsymbol{C}$ and $\boldsymbol{D}$ indicate transfected cells. Endog., Endogenous.

construct (GFP-SLAIN2-N1). The expression of this SLAIN2 fragment, which can bind to ch-TOG but not to EBs, CLIPs, or CLASPs, has previously been shown to disrupt MT plus-end recruitment of ch-TOG and inhibit MT growth similar to SLAIN2 knockdown in HeLa cells (van der Vaart et al., 2011).

Disruption of the SLAIN-ch-TOG complex in DIV1 neurons by transfection of ch-TOG shRNA or GFP-SLAIN2-N1 construct strongly affected the number of growing MT ends as observed by immunofluorescent labeling of DIV5 neurons with EB1 antibody (Fig. $2 B-D$ ). These results are similar to those seen in nonneuronal cells after SLAIN2 or ch-TOG depletion (van der Vaart et al., 2011). Knock down of SLAIN1 and SLAIN2 had a much milder effect, most likely because it was less profound (data not shown).

Next, we set out to investigate MT dynamics in neuronal cells after disruption of the SLAIN-ch-TOG complex. We used as a marker of growing MT ends the dimeric version of the C-terminal 43-aa-long MT plus-end tracking fragment of the spectraplakin MACF2 [MACF43-LZ (Honnappa et al., 2009)], which we named mCherry-MT+TIP. Plus-end tracking behavior of mCherryMT+ TIP could be readily observed in control GFP-expressing cells as well as in cells expressing GFP-SLAIN2-N1, SLAIN1/2, or chTOG shRNAs (Fig. 3A). However, when the SLAIN-ch-TOG complex was disrupted, persistent MT growth was diminished due to a strongly increased frequency of catastrophes (Fig. $3 B, C$ ). This effect was similar in all neuronal compartments, including cell bodies, axons, dendrites, and axonal growth cones (data not shown). Instan- taneous MT growth rate was reduced in ch-TOG depleted cells and in cells expressing GFP-SLAIN2-N1 (Fig. 3D), in line with the role of ch-TOG as a MT polymerization-promoting factor. SLAIN1/2 depletion had no effect (Fig. 3D), likely because the knockdown was incomplete. It should be noted that fast growth episodes were still observed in all conditions (Fig. 3D), indicating that MT polymerization rate is less sensitive to reduction in SLAIN and ch-TOG levels than growth processivity. In conclusion, the disruption of the SLAIN-ch-TOG complex in neuronal cells perturbs persistent MT growth.

\section{SLAIN and ch-TOG play a role in axon extension}

To assess the morphological effect of disrupting MT dynamics by inhibiting SLAIN-ch-TOG complex formation, we analyzed the length of axons and dendrites at DIV 5 in neurons that were transfected with different shRNAs or GFP-SLAIN2-N1 at DIV1. Immunofluorescent staining with antibodies against tau and MAP2 was used to distinguish axons and dendrites (Fig. 4A). Depletion of SLAIN1/2 or ch-TOG and the overexpression of GFPSLAIN2-N1 caused a similar, significant decrease in axonal length at DIV5 compared with control cells (Fig. 4B,C). Dendritic length was highly variable at this developmental stage and showed no significant differences (Fig. 4C). From these experiments, we conclude that MT growth dynamics regulated by the SLAIN-ch-TOG complex is important for proper axon outgrowth during development of primary hippocampal rat neurons. 

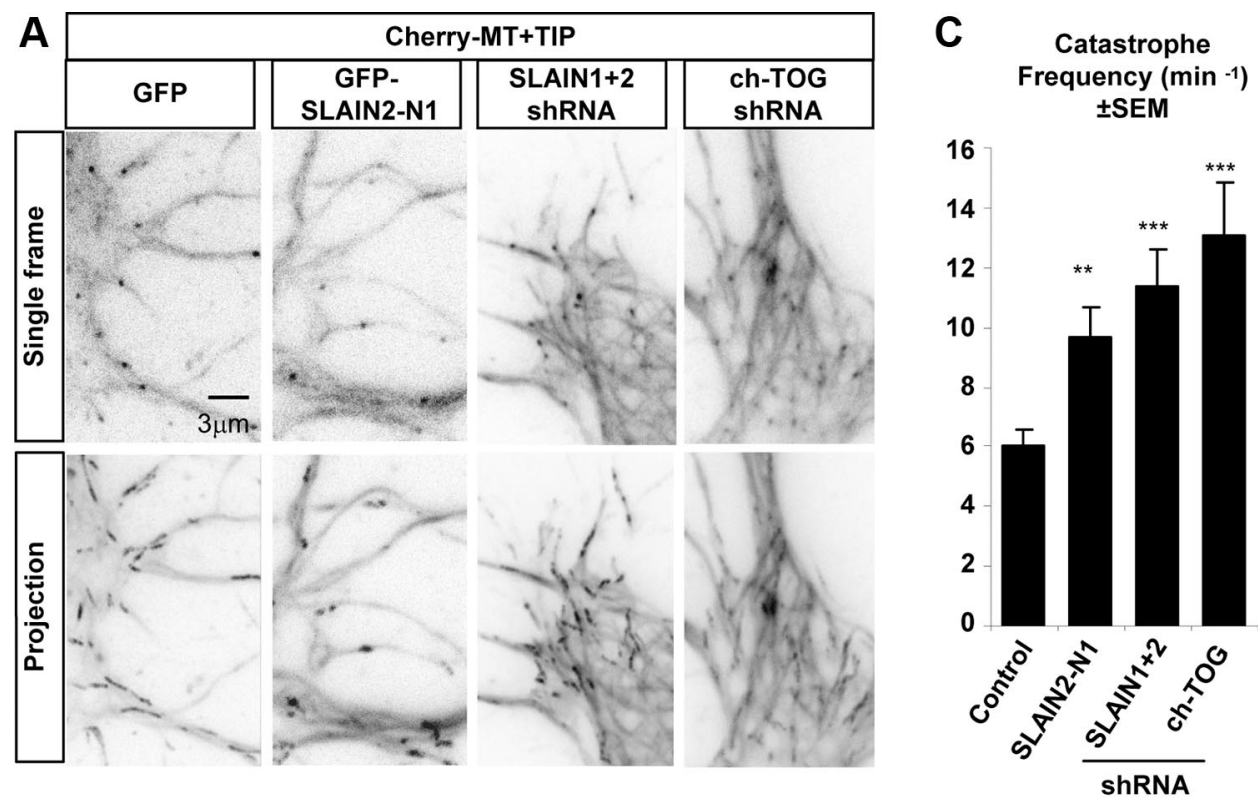

B

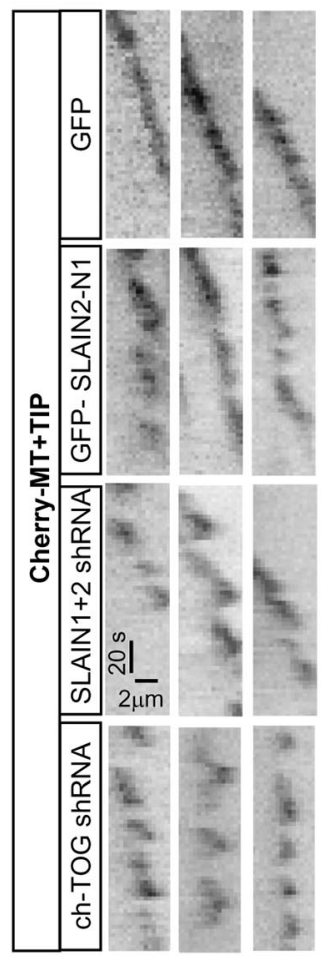

D Instantaneous growth rate $(\mu \mathrm{m} / \mathrm{min})$
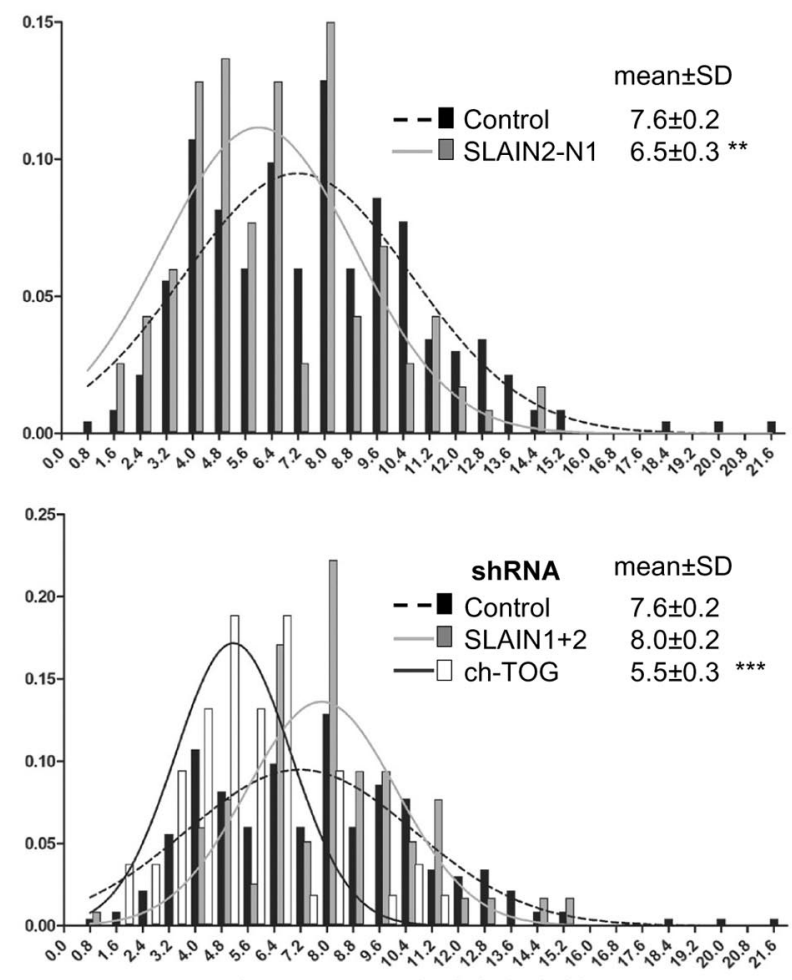

Figure 3. SLAIN-ch-TOG complex controls MT growth in neuronal cells.A, Liveimages of neurons transfected at DIV1 with mCherry-MT+TIP together with GFP, GFP-SLAIN2-N1, or shRNA constructs againstSLAIN1 and SLAIN2 orch-TOG. Live images were collected at DIV5 with 0.5 sintervals. Singleframes and maximum projections of 61 frames (bottom) areshown. B, Kymographs illustrating MT growth using mChemy-MT + TIP in neurons coexpressing mCherry-MT + TIP together with GFP (control), GFP-SLAIN2-N1, SLAIN1 + 2, orch-TOG shRNAs.C, Quantification of the catastrophefrequency in control, GFP-SLAIN2-N1-expressing cells, or cells expressing the indicated shRNAs using data shown in $A$ and $B$. Error bars indicate SEM. Fifty to 200 growth episodes in fiveto nine cells observed predominantly in the cell body and proximal neurites were analyzed in each condition. Data for GFP control and controlshRNA showed nosignificant differences and werepooled. D, Mean instantaneous growthrates (growthrates measured between two framesacquired with a 0.5 sinterval) and histograms of MT growth rate distributions based on the displacement of mCherry-MT + TIP comets in DIV5 neurons cotransfected at DIV1 with GFP (control), GFP-SLAIN2-N1, or the shRNA constructs. Values significantly different from control are indicated by asterisks $\left({ }^{* *} p<0.05,{ }^{* * *} p<0.001\right)$.

\section{Discussion}

In this study, we used postmitotic neurons to analyze the expression and role of ch-TOG and SLAIN1/2, factors previously shown to be critically important for proper MT growth in dividing cells (van der Vaart et al., 2011). We found that ch-TOG and SLAIN1/2 are expressed throughout mouse brain development and in primary hippocampal neuron cultures. Disruption of the SLAIN-ch-TOG complex by ch-TOG depletion or overexpression of the dominant-negative SLAIN2 reduced the number of EB1 comets in neuronal cell bodies and increased MT catastrophe frequency in cell bodies, axons, and dendrites. Simultaneous depletion 

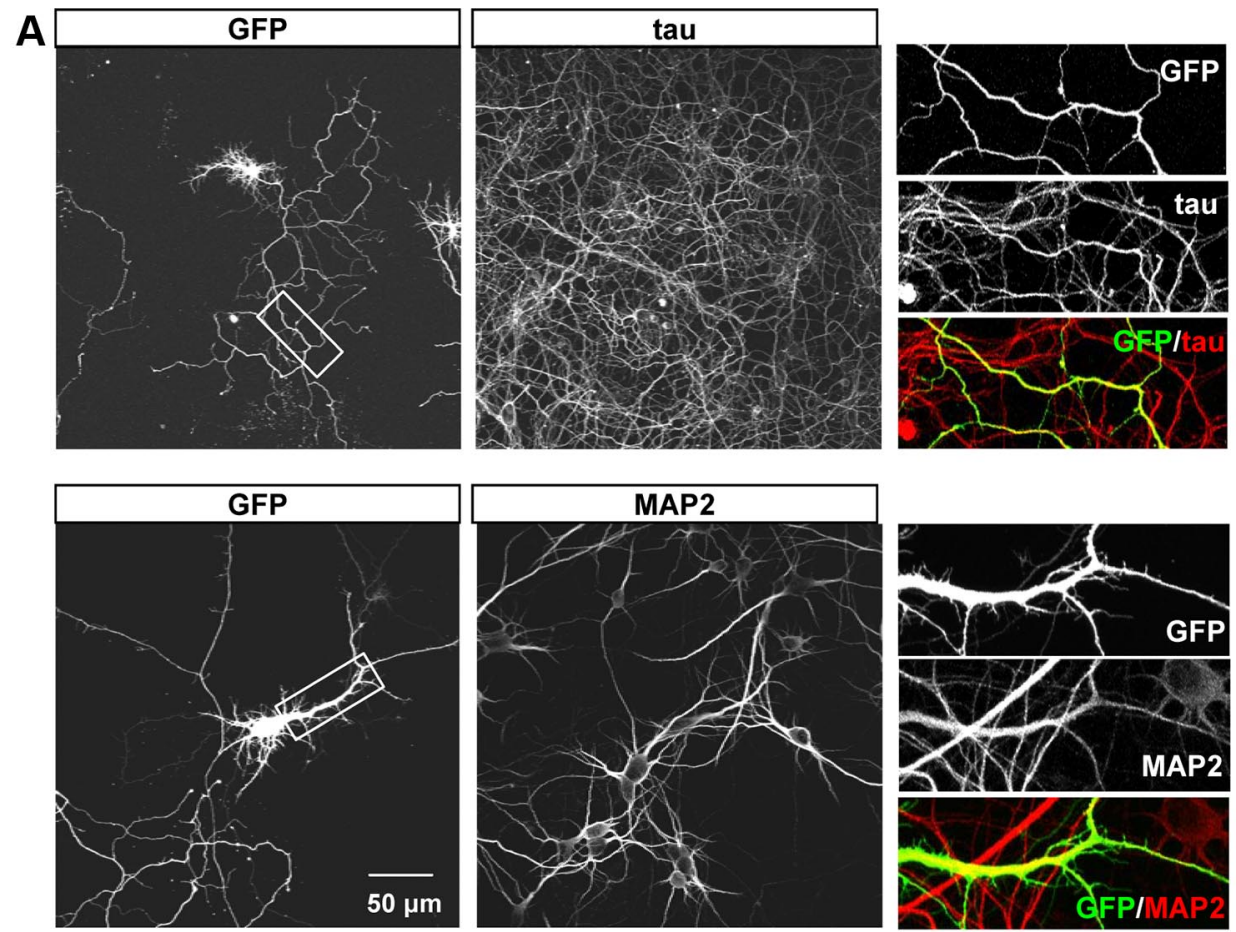

B
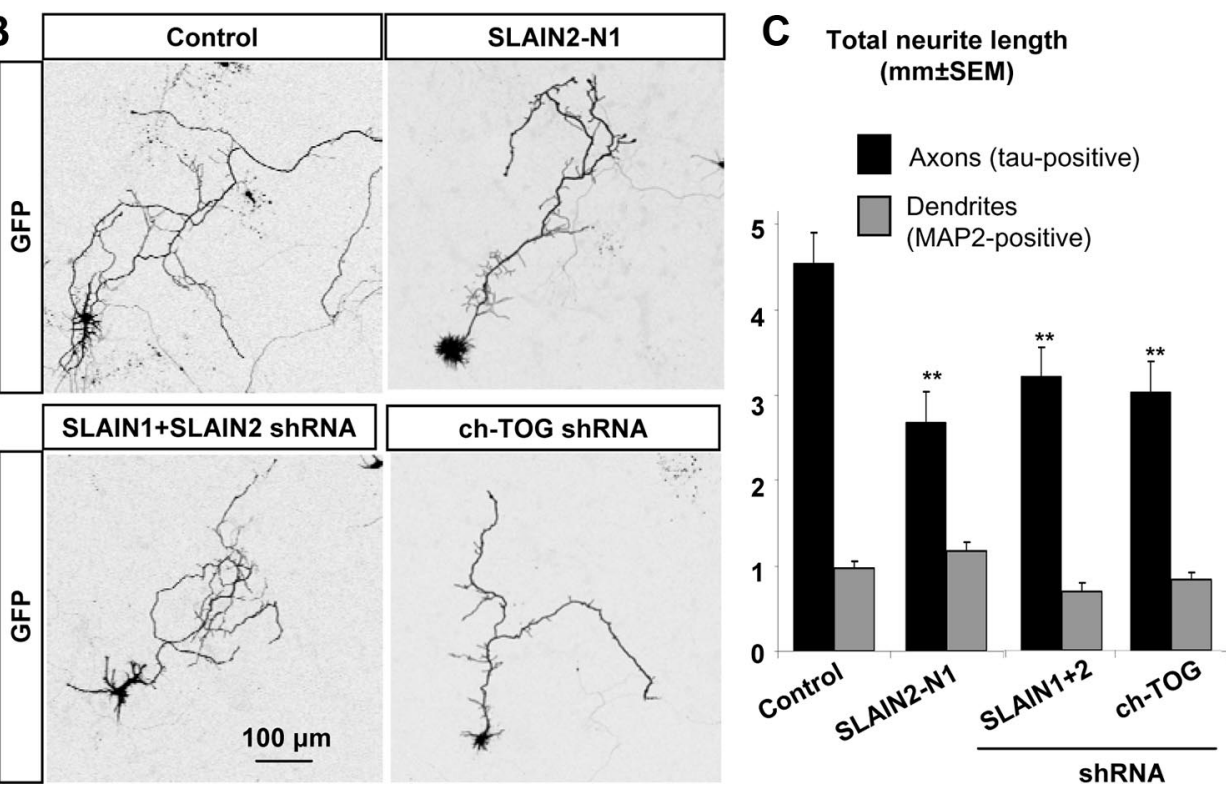

Figure 4. ch-TOG and SLAIN1/2 are necessary for axon extension. $A$, Images of neurons transfected at DIV1 with the control GFP construct, fixed at DIV5, and labeled with tau or MAP2 antibodies. $B$, Images of neurons transfected with the indicated constructs and GFP as a neuronal morphology marker at DIV1 and fixed at DIV5. C, Quantification of the total length of axons and dendrites in DIV5 neurons transfected at DIV1 with the indicated constructs. GFP was used as a morphology marker, and tau and MAP2 staining were used to distinguish axons and dendrites. Nine to 12 cells were analyzed for each condition. Statistically significant differences are indicated $\left.{ }^{* *} p<0.05\right)$. Error bars indicate SEM.

of SLAIN1 and SLAIN2 also greatly increased the frequency of catastrophes, although it had no strong effect on the number of EB1 comets, most likely because it was less efficient. These results indicate that the SLAIN-ch-TOG complex promotes processive MT polymerization in all neuronal compartments.

In nonneuronal cells, SLAIN1/2 and ch-TOG together with EBs help support frequent and persistent MT outgrowth, which is needed to maintain a radial MT array necessary for proper localization of membrane compartments and cell polarity (van der Vaart et al., 2011). In neurons, SLAIN1/2 and ch-TOG have a similar role: when the SLAIN-ch-TOG complex is disrupted, MT growth episodes become less frequent and are more often interrupted by catastrophes. This change in MT dynamics is expected to have a negative effect on the invasion of lamellipodia by MTs and MT stabilization and elongation, processes critical for neurite initiation and extension (Conde and Cáceres, 2009; Hoogenraad and Bradke, 2009; Poulain and Sobel, 2010). The disruption of the SLAIN-ch-TOG complex in the first days of neuronal development (DIV1-DIV5) led to a reduction of axonal length. This is in line with the data obtained using MT-targeting drugs: application of low doses of nocodazole, vin- 
blastine, or taxol has been shown to slow down the axonal growth cone propagation (Tanaka et al., 1995; Yu and Baas, 1995; Rochlin et al., 1996; Williamson et al., 1996; Gallo and Letourneau, 1999). In our experiments, axon extension was reduced but not abolished, most likely due to the timing of our inhibitory treatments (transfection at DIV1) and the incomplete disruption of SLAIN1/2 and chTOG function. Interestingly, the extent of reduction of axon length in our experiments was similar to that observed in another study where hippocampal neurons were treated with low doses of nocodazole from DIV1 to DIV3 (Witte et al., 2008). Dendrite extension was not significantly affected, most likely because, in contrast to axons, dendrites do not undergo extensive elongation at the developmental stage examined (DIV5) and are therefore likely less sensitive to alterations in MT dynamics.

Recently, a general model of cell elongation showed that cell length can be controlled by parameters of MT growth dynamics, such as polymerization velocity and catastrophe frequency (Picone et al., 2010). Our results are in agreement with this model because they show that an increase in MT catastrophe frequency leads to shorter MT-dependent cell extensions, axons of developing neurons.

The enrichment of ch-TOG and SLAIN1/2 in neuronal growth cones suggests a role of this complex in growth cone dynamics similar to the roles of CLIPs and CLASPs (Lee et al., 2004; Neukirchen and Bradke, 2011). These protein families can interact with SLAINs (van der Vaart et al., 2011), and it remains to be determined whether SLAINs play a role in the accumulation of these proteins at the MT tips in growth cones. Interestingly, in Drosophila, the ch-TOG homolog Mini spindles interacts with the CLASP homolog and can antagonize its function in axon guidance (Lowery et al., 2010). In vertebrate cells, CLASPs can both impede and promote axonal growth depending on whether they bind to the MT lattice or the growing MT tips (Lee et al., 2004; Hur et al., 2011), suggesting that they can either cooperate with ch-TOG or antagonize its axon growth-promoting activity.

Together, our study has identified the SLAIN-ch-TOG MT tip complex as an important regulator of axon growth in primary hippocampal neurons, indicating that basic MT polymerization machinery is important for a complex differentiation process such as neuronal development.

\section{References}

Akhmanova A, Steinmetz MO (2008) Tracking the ends: a dynamic protein network controls the fate of microtubule tips. Nat Rev Mol Cell Biol 9:309-322. CrossRef Medline

Baas PW (1999) Microtubules and neuronal polarity: lessons from mitosis. Neuron 22:23-31. CrossRef Medline

Banker G, Goslin K (1988) Developments in neuronal cell culture. Nature 336:185-186. CrossRef Medline

Charrasse S, Mazel M, Taviaux S, Berta P, Chow T, Larroque C (1995) Characterization of the cDNA and pattern of expression of a new gene overexpressed in human hepatomas and colonic tumors. Eur J Biochem 234: 406-413. CrossRef Medline

Charrasse S, Coubes P, Arrancibia S, Larroque C (1996) Expression of the tumor over-expressed ch-TOG gene in human and baboon brain. Neurosci Lett 212:119-122. CrossRef Medline

Charrasse S, Schroeder M, Gauthier-Rouviere C, Ango F, Cassimeris L, Gard DL, Larroque C (1998) The TOGp protein is a new human microtubule-associated protein homologous to the Xenopus XMAP215. J Cell Sci 111:1371-1383. Medline

Conde C, Cáceres A (2009) Microtubule assembly, organization and dynamics in axons and dendrites. Nat Rev Neurosci 10:319-332. CrossRef Medline

Gallo G, Letourneau PC (1999) Different contributions of microtubule dynamics and transport to the growth of axons and collateral sprouts. J Neurosci 19:3860-3873. Medline

Hirst CE, Ng ES, Azzola L, Voss AK, Thomas T, Stanley EG, Elefanty AG
(2006) Transcriptional profiling of mouse and human ES cells identifies SLAIN1, a novel stem cell gene. Dev Biol 293:90-103. CrossRef Medline Hirst CE, Lim SM, Pereira LA, Mayberry RA, Stanley EG, Elefanty AG (2010) Expression from a betageo gene trap in the Slain 1 gene locus is predominantly associated with the developing nervous system. Int J Dev Biol 54:1383-1388. CrossRef Medline

Honnappa S, Gouveia SM, Weisbrich A, Damberger FF, Bhavesh NS, Jawhari H, Grigoriev I, van Rijssel FJ, Buey RM, Lawera A, Jelesarov I, Winkler FK, Wüthrich K, Akhmanova A, Steinmetz MO (2009) An EB1-binding motif acts as a microtubule tip localization signal. Cell 138:366-376. CrossRef Medline

Hoogenraad CC, Bradke F (2009) Control of neuronal polarity and plasticity-a renaissance for microtubules? Trends Cell Biol 19:669-676. CrossRef Medline

Hoogenraad CC, Milstein AD, Ethell IM, Henkemeyer M, Sheng M (2005) GRIP1 controls dendrite morphogenesis by regulating EphB receptor trafficking. Nat Neurosci 8:906-915. Medline

Howard J, Hyman AA (2007) Microtubule polymerases and depolymerases. Curr Opin Cell Biol 19:31-35. CrossRef Medline

Hur EM, Saijilafu, Lee BD, Kim SJ, Xu WL, Zhou FQ (2011) GSK3 controls axon growth via CLASP-mediated regulation of growth cone microtubules. Genes Dev 25:1968-1981. CrossRef Medline

Jaworski J, Kapitein LC, Gouveia SM, Dortland BR, Wulf PS, Grigoriev I, Camera P, Spangler SA, Di Stefano P, Demmers J, Krugers H, Defilippi P, Akhmanova A, Hoogenraad CC (2009) Dynamic microtubules regulate dendritic spine morphology and synaptic plasticity. Neuron 61:85-100. CrossRef Medline

Lee H, Engel U, Rusch J, Scherrer S, Sheard K, Van Vactor D (2004) The microtubule plus end tracking protein Orbit/MAST/CLASP acts downstream of the tyrosine kinase $\mathrm{Abl}$ in mediating axon guidance. Neuron 42:913-926. CrossRef Medline

Lowery LA, Lee H, Lu C, Murphy R, Obar RA, Zhai B, Schedl M, Van Vactor D, Zhan Y (2010) Parallel genetic and proteomic screens identify Msps as a CLASP-Abl pathway interactor in Drosophila. Genetics 185:1311-1325. CrossRef Medline

Nakagawa H, Koyama K, Murata Y, Morito M, Akiyama T, Nakamura Y (2000) EB3, a novel member of the EB1 family preferentially expressed in the central nervous system, binds to a CNS-specific APC homologue. Oncogene 19:210-216. CrossRef Medline

Nakagawa T, Hoogenraad CC (2011) Lentiviral transgenesis. Methods Mol Biol 693:117-142. CrossRef Medline

Neukirchen D, Bradke F (2011) Cytoplasmic linker proteins regulate neuronal polarization through microtubule and growth cone dynamics. J Neurosci 31:1528-1538. CrossRef Medline

Picone R, Ren X, Ivanovitch KD, Clarke JD, McKendry RA, Baum B (2010) A polarised population of dynamic microtubules mediates homeostatic length control in animal cells. PLoS Biol 8:e1000542. CrossRef Medline

Poulain FE, Sobel A (2010) The microtubule network and neuronal morphogenesis: dynamic and coordinated orchestration through multiple players. Mol Cell Neurosci 43:15-32. CrossRef Medline

Rochlin MW, Wickline KM, Bridgman PC (1996) Microtubule stability decreases axon elongation but not axoplasm production. J Neurosci 16: 3236-3246. Medline

Rubinson DA, Dillon CP, Kwiatkowski AV, Sievers C, Yang L, Kopinja J, Rooney DL, Zhang M, Ihrig MM, McManus MT, Gertler FB, Scott ML, Van Parijs L (2003) A lentivirus-based system to functionally silence genes in primary mammalian cells, stem cells and transgenic mice by RNA interference. Nat Genet 33:401-406. CrossRef Medline

Schmitz SK, Hjorth JJ, Joemai RM, Wijntjes R, Eijgenraam S, de Bruijn P, Georgiou C, de Jong AP, van Ooyen A, Verhage M, Cornelisse LN, Toonen RF, Veldkamp W (2011) Automated analysis of neuronal morphology, synapse number and synaptic recruitment. J Neurosci Methods 195:185-193. CrossRef Medline

Schuyler SC, Pellman D (2001) Microtubule "plus-end-tracking proteins": the end is just the beginning. Cell 105:421-424. CrossRef Medline

Tanaka E, Ho T, Kirschner MW (1995) The role of microtubule dynamics in growth cone motility and axonal growth. J Cell Biol 128:139-155. CrossRef Medline

van der Vaart B, Manatschal C, Grigoriev I, Olieric V, Gouveia SM, Bjelic S, Demmers J, Vorobjev I, Hoogenraad CC, Steinmetz MO, Akhmanova A (2011) SLAIN2 links microtubule plus end-tracking proteins and controls microtubule growth in interphase. J Cell Biol 193:1083-1099. CrossRef Medline 
Williamson T, Gordon-Weeks PR, Schachner M, Taylor J (1996) Microtubule reorganization is obligatory for growth cone turning. Proc Natl Acad Sci U S A 93:15221-15226. CrossRef Medline

Witte H, Neukirchen D, Bradke F (2008) Microtubule stabilization specifies initial neuronal polarization. J Cell Biol 180:619-632. CrossRef Medline
Yu W, Baas PW (1995) The growth of the axon is not dependent upon net microtubule assembly at its distal tip. J Neurosci 15:6827-6833. Medline 